\title{
NSAR oder Triptane?
}

Fragestellung: Wie gut wirken nicht steroidale Antirheumatika und Triptane zur Behandlung akuter Migräneattacken?

Hintergrund: Leichte und mittelschwere Migräneattacken werden nach den Leitlinien der Deutschen Gesellschaft für Neurologie (DGN) mit nicht steroidalen Antirheumatika (NSAR) oder Acetylsalicylsäure (ASS) behandelt. Bei Migräneattacken, die auf diese Therapie nicht ausreichend ansprechen, oder wenn die Medikamente nicht gut vertragen werden, kommen Triptane zum Einsatz. In der Zwischenzeit gibt es viele randomisierte Studien, welche die einzelnen Substanzen entweder gegen Placebo oder gegeneinander untersuchen. Die Autoren aus China haben aus diesem Grund eine systematische Literaturrecherche und eine Netzwerk-Metaanalyse durchgeführt.

Patienten und Methodik: In die Metaanalyse gingen insgesamt 88 randomisierte Studien ein. Unter anderem wurden die Schmerzfreiheit nach zwei Stunden und Nebenwirkungen ausgewertet. Erfasst wurden ASS und Ibuprofen, Diclofenac sowie die Triptane Almotriptan, Eletriptan, Naratriptan, Rizatriptan, Sumatriptan und Zolmitriptan.

Ergebnisse: Alle Analgetika und Triptane sind einer Behandlung mit Placebo bezüglich des Outcomes Schmerzfreiheit nach zwei Stunden überlegen. Die 95\%-Konfidenzintervalle der Wirkung bezüglich Kopfschmerzfreiheit nach zwei Stunden überlappen sich. Numerisch sind Rizatriptan und Eletriptan am besten wirksam. Die Wirkung der Triptane ist mit der von Diclofenac, Ibuprofen und ASS vergleichbar. Bei den Nebenwirkungen schneiden Ibuprofen, Eletriptan und Diclofenac am besten ab. Die höchsten Nebenwirkungsraten haben ASS und Naratriptan.

Xu H, Han W, Wang J et al. Network meta-analysis of migraine disorder treatment by NSAIDs and triptans. J Headache Pain 2016; 17: 113
Schlussfolgerungen: In einer Netzwerk-Metaanalyse von 88 Studien hatte Eletriptan die beste Wirksamkeit und Ibuprofen die beste Verträglichkeit bei der Behandlung akuter Migräneattacken.

\section{- Kommentar von Hans-Christoph Diener, Essen}

\section{ASS, NSAR und Triptane vergleichbar wirksam}

Metaanalysen sind eine gute wissenschaftliche Methode, um die Wirksamkeit und Verträglichkeit neuer Therapien zu vergleichen, wenn es eine unübersehbar große Zahl an Vergleichsstudien gibt. Der Vergleich von ASS, NSAR und Triptanen zeigt, dass sie bezüglich ihrer Wirksamkeit ähnlich sind. Tendenziell am besten wirksam sind Eletriptan und Rizatriptan. Alle Migränemittel werden relativ gut vertragen. Das beste Verträglichkeitsprofil hat Ibuprofen. Die Ergebnisse der Metaanalyse entbinden den behandelnden Arzt allerdings nicht davon, für den einzelnen Patienten die Substanz herauszufinden, die das beste Verhältnis von Wirksamkeit bezüglich Schmerzfreiheit nach einer oder nach zwei Stunden und Verträglichkeit hat. Erschwerend kommt hinzu, dass bei den meisten Triptanen in der Zwischenzeit der Patentschutz abgelaufen ist und sie damit preiswert zu verschreiben sind, während Eletriptan immer noch patentgeschützt ist. Die fixe Kombination aus Sumatriptan und Naproxen ist einer Monotherapie mit Triptanen nicht überlegen, hat jedoch die kombinierten Nebenwirkungen beider Substanzen. 